\title{
Lifestyle-integrated functional exercise to prevent falls and promote physical activity: Results from the LiFE-is-LiFE randomized non-inferiority trial
}

\author{
Carl-Philipp Jansen ${ }^{1,2}$, Corinna Nerz², Sarah Labudek ${ }^{1}$, Sophie Gottschalk³ , Franziska Kramer-Gmeiner ${ }^{1}$, \\ Jochen Klenk ${ }^{2,4,5}$, Judith Dams ${ }^{3}$, Hans-Helmut König ${ }^{3}$, Lindy Clemson ${ }^{6}$, Clemens Becker $^{2}$ and \\ Michael Schwenk ${ }^{*}$ (D)
}

\begin{abstract}
Background: The 'Lifestyle-integrated Functional Exercise' (LiFE) program successfully reduced risk of falling via improvements in balance and strength, additionally increasing physical activity (PA) in older adults. Generally being delivered in an individual one-to-one format, downsides of LiFE are considerable human resources and costs which hamper large scale implementability. To address this, a group format (gLiFE) was developed and analyzed for its noninferiority compared to LiFE in reducing activity-adjusted fall incidence and intervention costs. In addition, PA and further secondary outcomes were evaluated.
\end{abstract}

Methods: Older adults (70 + years) at risk of falling were included in this multi-center, single-blinded, randomized non-inferiority trial. Balance and strength activities and means to enhance PA were delivered in seven intervention sessions, either in a group (gLiFE) or individually at the participant's home (LiFE), followed by two "booster" phone calls. Negative binomial regression was used to analyze non-inferiority of gLiFE compared to LiFE at 6-month followup; interventions costs were compared descriptively; secondary outcomes were analyzed using generalized linear models. Analyses were carried out per protocol and intention-to-treat.

Results: Three hundred nine persons were randomized into gLiFE $(n=153)$ and LiFE $(n=156)$. Non-inferiority of the incidence rate ratio of gLiFE was inconclusive after 6 months according to per protocol (mean $=1.27 ; 95 \% \mathrm{Cl}$ : 0.80; 2.03) and intention-to-treat analysis (mean $=1.18 ; 95 \% \mathrm{Cl}: 0.75 ; 1.84$ ). Intervention costs were lower for gLiFE compared to LiFE ( $€ 121$ under study conditions; - $€ 212 €$ under "real world" assumption). Falls were reduced between baseline and follow-up in both groups (gLiFE: -37\%; LiFE: -55\%); increases in PA were significantly higher in gLiFE (+ 880 steps; 95\% Cl 252; 1,509). Differences in other secondary outcomes were insignificant.

Conclusions: Although non-inferiority of gLiFE was inconclusive, gLiFE constitutes a less costly alternative to LiFE and it comes with a significantly larger enhancement of daily PA. The fact that no significant differences were found in any secondary outcome underlines that gLiFE addresses functional outcomes to a comparable degree as LiFE.

*Correspondence: schwenk@nar.uni-heidelberg.de

${ }^{1}$ Network Aging Research, Heidelberg University, Bergheimer Strasse 20, 69115 Heidelberg, Germany

Full list of author information is available at the end of the article permits use, sharing, adaptation, distribution and reproduction in any medium or format, as long as you give appropriate credit to the original author(s) and the source, provide a link to the Creative Commons licence, and indicate if changes were made. The images or other third party material in this article are included in the article's Creative Commons licence, unless indicated otherwise in a credit line to the material. If material is not included in the article's Creative Commons licence and your intended use is not permitted by statutory regulation or exceeds the permitted use, you will need to obtain permission directly from the copyright holder. To view a copy of this licence, visit http://creativecommons.org/licenses/by/4.0/. The Creative Commons Public Domain Dedication waiver (http://creativeco mmons.org/publicdomain/zero/1.0/) applies to the data made available in this article, unless otherwise stated in a credit line to the data. 
Advantages of both formats should be evaluated in the light of individual needs and preferences before recommending either format.

Trial registration: The study was preregistered under clinicaltrials.gov (identifier: NCT03462654) on March $12^{\text {th }} 2018$

Keywords: Fall prevention, Non-inferiority trial, Fall risk, Intervention costs, Physical activity promotion

\section{Background}

Tackling physical inactivity and mobility disability in the face of wide-spread sedentariness has been declared a paramount objective which is founded on the numerous positive effects on health and agingassociated morbidity in older persons [1, 2]. Physical activity (PA) further improves independence, increases participation, and enhances quality of life [3, 4]. However, PA comes with certain risks. Every transition and step increase the risk of falling, which holds true particularly when performed unsafely by older adults with low functional capacity [5-8]. Recommendations to increase PA in older persons have often neglected this potential trade-off. Especially walking has been recognized as a "hazardous" PA in older persons $[9,10]$. Arguably, beneficial effects of high PA may outweigh this risk by maintenance or improvement of functional capacity and mobility in the midterm, but oversimplified recommendations for increasing PA may not be unconditionally appropriate for the older population. Novel interventions should therefore be tested looking at an increase of safe PA, being defined as, e.g., falls per one million steps or falls per distance walked [11]. This requires sensor-based measurements of PA and mobility alongside classical outcome assessments of functional performance and perceived function. In summary, interventions should increase PA and simultaneously prevent falls and fall-related injuries. Examinations of a combined endpoint of fall risk and activity have been proposed as the gold standard approach [11-13].

The 'Lifestyle-integrated Functional Exercise' (LiFE) trial is a landmark study in this respect, having looked at both PA and falls [14], although without combining these endpoints for analysis. The study had shown significant improvement of balance and strength capacity of older persons aged $70+$ years and further promoted an increase of PA [15]. A possible downside of LiFE in certain settings is that it is delivered individually by therapists in seven home visits. This comes with considerable human resources and costs. A smaller pilot trial from Canada raised the idea that a group-based LiFE format may be similarly effective [16]. This was supported by findings from a feasibility study evaluating the groupbased LiFE (gLiFE) program used in this trial [17]. The economical assumption, that a group format could be less costly, as well as the group format's effectiveness in terms of the abovementioned combined endpoint have yet to be investigated.

Therefore, a non-inferiority trial was carried out to evaluate whether a group-based LiFE (gLiFE) program is not less effective than the original LiFE program (LiFE) by more than an acceptable amount while being less costly in terms of intervention costs. The acceptable amount is a predefined non-inferiority margin for the treatment effect in the trial's primary outcome [18]. Noninferiority investigations require that the reference treatment's efficacy is established [18]. Given the high quality of the LiFE trial and the positive effects found [14], we considered this prerequisite confirmed.

Primary objectives of this study were 1) to compare non-inferiority of gLiFE compared to LiFE in reducing activity-adjusted fall incidence; and 2) to compare intervention costs of both formats. The corresponding hypotheses were that gLiFE is not less efficacious than LiFE in reducing activity-adjusted fall incidence, and that its delivery is less costly compared to LiFE. The secondary objective was to compare effectiveness of both formats regarding functional (dis-)ability, adherence, motor capacity, fall-related outcomes, fear of falling, balance confidence, and adverse events.

\section{Methods}

Study design

This study ("LiFE-is-LiFE") was a multi-center, singleblinded, randomized non-inferiority trial conducted in Heidelberg and Stuttgart, Germany. The full study protocol is available elsewhere [19]. The study was preregistered under clinicaltrials.gov (identifier: NCT03462654) on March $12^{\text {th }} 2018$. Reporting in this article is aligned with the CONSORT extension in non-inferiority trials [18] [see CONSORT checklist, Additional file 3].

In addition to baseline assessment, follow-up assessments were carried out six and twelve months after intervention start (reference was the date of the first ( $\mathrm{g}$ )LiFE session), with a tolerance of \pm 2 weeks.

\section{Participants and eligibility criteria}

For recruitment purposes a list of all persons aged $70+$ was drawn from municipality registries in both cities. Persons were drawn consecutively in waves of between 250 and 1.000 persons and contacted between April 
2018 and July 2019 by mail. If interested, participants could contact the study sites for a first eligibility screening by telephone. In case of a positive telephone screening, an inhouse screening was scheduled. Participant flow is depicted in Fig. 1. To be included in the study, participants had either a) experienced at least one injurious or more than one non-injurious fall in the year prior to study participation according to self-report, or b) were designated as having high risk of falls when indicating self-perceived balance decline and needing $\geq 12 \mathrm{~s}$ for the "Timed Up-and-Go" (TUG) [20] test. Those who already exercised more than once per week or indicated to carry out more than 150 min of moderate to vigorous PA per week were excluded. A detailed list of further exclusion criteria is provided in the study protocol [19].

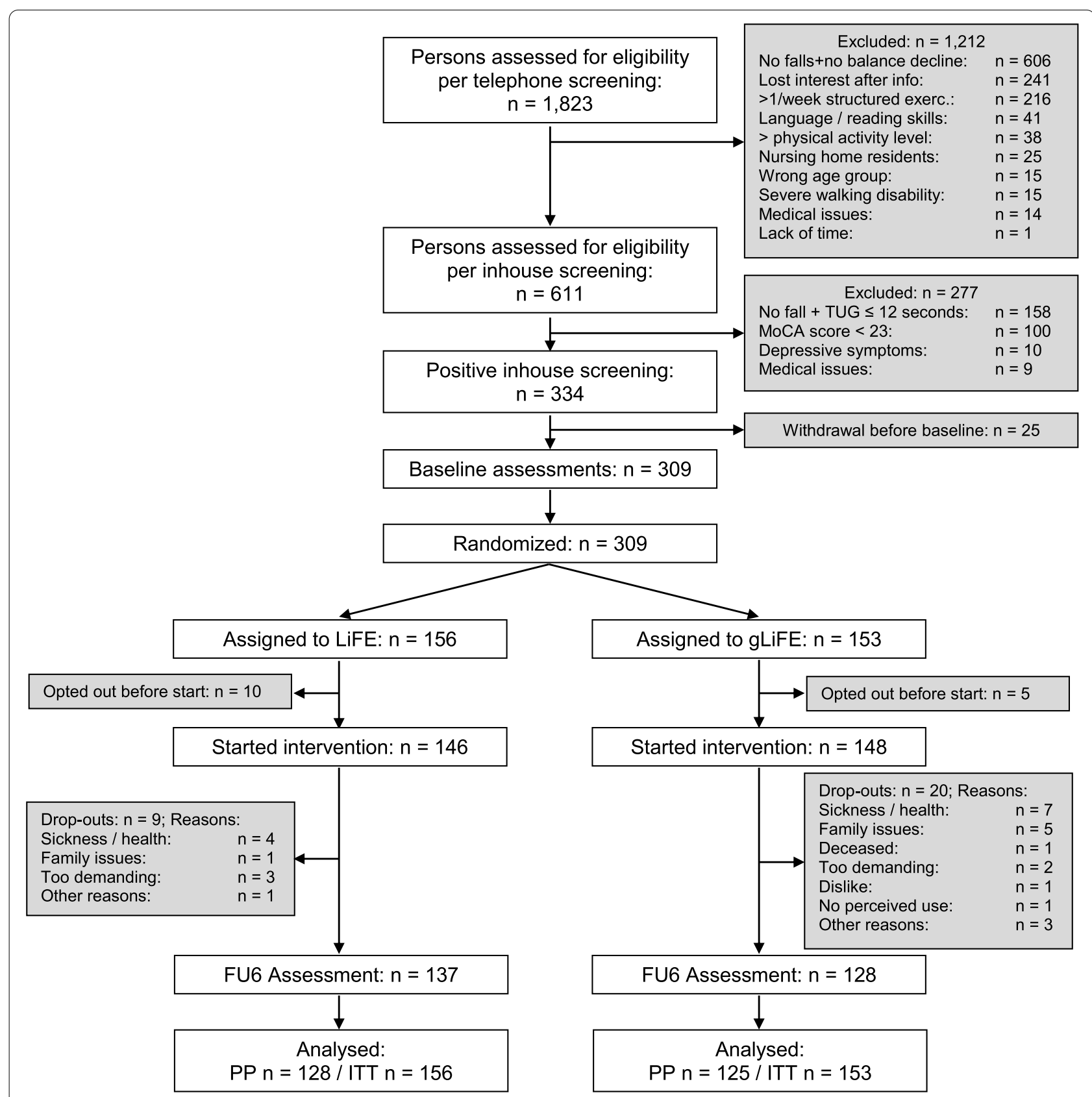

Fig. 1 Participant flow; FU6: 6-month follow-up assessment; PP: per protocol; ITT: intention-to-treat; MoCA: Montreal Cognitive Assessment; TUG: Timed Up-and-Go 


\section{Randomization and blinding}

Participants were randomized after baseline assessment into one of the two intervention arms through block-randomization. Apart from block sizes, randomization was concealed, i.e., staff was not aware of the sequence before randomization. Randomization and group assignment was carried out by the study site coordinators $(\mathrm{CPJ}, \mathrm{CN})$ in an externally managed database without possibility to alter group allocation afterwards. In case of withdrawal from intervention, participants were still eligible for follow-up assessments. Assessors were blinded to group allocation at all times.

\section{Intervention programs}

A detailed description including a TIDieR checklist of both intervention formats is included in the study protocol [19]. In the LiFE program, balance and strength activities as well as general PA promoting activities are embedded into everyday tasks and routines, with the overall aim to integrate them in a way that these activities can be performed multiple times a day [14]. As there was no standardized group format of the LiFE program available, gLiFE had been developed according to Medical Research Council guidelines [21] and piloted in advance to the intervention start [17]. In both intervention arms, intervention components were taught in accordance with the LiFE trainer's manual [15], including strength and balance activities as well as strategies to enhance physically active behavior and to habitualize activities as part of individual daily routine. LiFE and gLiFE were delivered in seven sessions within eleven weeks, either in a group (gLiFE) or at the participant's home (LiFE), followed by two booster phone calls in week four and ten after the last intervention session. During the intervention sessions a total of seven balance activities, seven strength activities for the lower extremities, and two PA promoting activities were delivered. To help participants establish a LiFE routine as part of their daily life, they learned how to independently select, execute, and adapt intensity of activities, and how to identify appropriate daily situations in which LiFE activities can be integrated. gLiFE group sessions were scheduled for two hours and held by two trainers with a maximum of 12 participants; LiFE sessions lasted approximately one hour and were delivered by one trainer. Trainers were either physio therapists, sports scientists, health psychologists, or occupational therapists who had attended a two-day workshop prior to the start of the intervention delivery, including a certification test.

\section{Outcomes}

\section{Primary outcomes}

Combined endpoint: Falls adjusted for PA. To measure PA, "activPAL4 $4^{\text {TM }}$ micro" accelerometers (PAL Technologies Ltd., Glasgow, Scotland) were attached to participants' central front thigh at baseline, 6-, and 12-month follow-up to continuously measure PA under "free-living" conditions for seven days ( $24 \mathrm{~h}$ ), i.e., activPALs were posted back to the respective study centers no earlier than the start of the ninth day of measurement. The sensor was wrapped in a nitrile finger cot fixed with a waterproof, adhesive, transparent film. The device has shown good to excellent reliability and validity [22]. If the device was removed earlier, data were used if at least two weekdays and the Sunday of the respective week were fully captured [23]. Given that walking activity can be seen as the most hazardous PA when it comes to risk of falling [9, 10], PA exposure was operationalized as mean steps/day.

Falls were defined as "an unexpected event in which the participant comes to rest on the ground, floor, or lower level" [24] and were recorded using a monthly falls calendar sent back by use of preaddressed and prestamped envelopes. In case of a fall, information on location, date, time, injuries, subsequent treatment related to the fall, and movement during which the person has fallen had to be provided on the calendar sheet. Following recommendations of Gillespie et al. [25], falls were followed-up via telephone calls to ascertain additional information and to determine the current health status of the person.

Intervention costs. Intervention costs were calculated as costs per participant for each group (LiFE/gLiFE). Personnel and material costs, trainers' and participants' travel expenses, and room rent were taken into account. The average group size of gLiFE sessions was 7.9 persons. The duration of the sessions (including time for travel and preparation) resulted in 1.8 (LiFE) and 3.0 (gLiFE) personnel hours per session. Personnel costs per hour were derived from the German wages agreement for civil services 2018 (“TVöD” salary level E13 and E10). Costs for materials, manuals, and working books were considered. Moreover, a room rent of $€ 50$ per day for the trainer workshop or per gLiFE session in one of the study centres was also taken into account. In the other study centre, a suitable room was available on site for the gLiFE sessions, therefore no room rent was incurred there.

Since study conditions deviate from conditions in case of an implementation in the "real world", interventions costs were also calculated for another scenario, based on assumptions that the project team considers to most realistically represent the implementation conditions. In this "real world" scenario, it was assumed that 20 trainers with a salary according to "TVöD" salary level E8 participate in the trainer workshop. It was assumed that 
on average 12 persons attend the gLiFE sessions and that each gLiFE trainer pair would conduct 12 training sessions per week, while one LiFE trainer could conduct 15 sessions in the same time. The duration of the LiFE/ gLiFE sessions (including time for travel and preparation) and phone calls were assumed to be $2.0 \mathrm{~h} / 2.5 \mathrm{~h}$ and $0.5 \mathrm{~h}$, respectively. For both interventions, no room rent was assumed. Furthermore, each trainer or trainer pair was assumed to have their own material set. The data and assumptions underlying the calculations of each scenario are summarized in an additional table [see Additional file 1].

\section{Secondary outcomes}

Physical activity. Mean steps/day were assessed to serve as offset variable in the primary outcome analysis to adjust falls for PA, and as PA outcome in itself.

Fall outcomes. Falls were assessed and defined according to Lamb et al. [24], that is, number of falls, fall rate per (half) person year, time to event (either fall or end of observation), number of fallers, and frequent fallers (i.e., more than one fall in the past six months). Fall consequences were categorized into minor, moderate or serious injuries according to a standardized system incorporating symptoms as well as medical care use [26].

Motor capacity. Gait performance was measured in terms of $7 \mathrm{~m}$ gait speed at comfortable and fast pace. The $30 \mathrm{~s}$ chair rise was used to evaluate functional leg strength [27]. Static balance was assessed using the adjusted eight level balance scale developed by Clemson et al. [14].

Functional (dis-)ability. The Late Life Function and Disability Instrument (LLFDI) was used to assess participants' difficulties in performing 32 different upper and lower extremity physical activities and actions as well performance of another 16 socially defined life tasks.

Adherence. We followed the consensus agreement by Hawley-Hague et al. [28] who recommend reporting adherence in terms of completion (attendance of at least $>75 \%$ of sessions is defined as completion [28]), attendance (percentage of sessions attended out of the actual number of sessions), and duration adherence (adherence to predefined LiFE activities at home, assessed using the Exercise Adherence Rating Scale (EARS) [29]). The EARS ranges from 0 to 24.

Fear of falling and balance confidence. Participants' fear of falling was assessed using the Short Falls Efficacy Scale-International [30], a self-rating scale including 7 items ranging from 'not at all concerned' (1 point) to 'very concerned' ( 4 points) and resulting in values between 7 ('not concerned about falling') and 28 points ('very concerned about falling'). The Activities-specific Balance Confidence Scale (ABC) was used to measure participants' confidence in maintaining their balance while performing certain daily activities.

For participants' characteristics, age, sex, body-mass index, number of medications, number of comorbidities, falls in the past six months, and cognitive status (Montreal Cognitive Assessment) were assessed.

\section{Sample size and non-inferiority margin}

Sample size was calculated based on 12 month data from the original LiFE study [14]; information on this calculation can be found in the study protocol [19]. As outlined in the limitations section COVID-19-induced changes have been made to the methods used for the present analyses. We used 6-month instead of 12-month data to determine non-inferiority of the primary outcome falls per PA. However, we kept the non-inferiority margin $(\Delta)$ as stated in the study protocol [19], that is, we accept a $20 \%$ difference in this outcome as a comparable reduction. As intervention costs of gLiFE are expected to be lower than of LiFE, no non-inferiority margin is defined for this outcome.

\section{Statistical analyses}

The analyses were carried out according to both the intention-to-treat principle (ITT) and the per-protocol principle (PP) to determine the robustness of the results due to missing values [31]. As dates were fixed for the gLiFE sessions, it was expected that some participants might be unable to attend all seven sessions. Therefore, attendance of a minimum of five sessions per participant was preset to assign participants to the PP sample. In accordance with the ITT principle, all randomized participants who completed baseline assessment were included, regardless of whether they had completed the intervention or prematurely dropped out of the study. In addition to missing information due to drop-out, there was occasional missing information in cases that otherwise completed the follow-up assessment. Overall, the percentage of missing values varied between 0 and $17 \%$ across different variables. As imputation of missing values is recommended for missing rates above 5\% [32], missing data were imputed using multiple imputation by chained equations (MICE) with predictive mean matching as imputation method [33]. In total, 10 datasets were created based on data from baseline and 6-months' follow-up assessments and analysed separately. Rubin's rules [34] were applied to pool results from each dataset.

Negative binomial regression was used to compare incidence rate ratios (IRR) of falls between gLiFE and LiFE,taking into account possible overdispersion. In the model for the combined endpoint-falls per PAmean steps/day were log-transformed and incorporated as exposure variable (offset). Confidence intervals 
for explorative comparison of changes between baseline and 6-month follow-up in secondary outcomes were obtained using a generalized linear model with repeated measures.

For the primary outcome, non-inferiority was indicated if the upper limit of the two-sided $95 \%$ confidence interval (CI) for gLiFE remained below the relative margin $(\Delta)$ of $20 \%$ from LiFE (IRR $=1.20$ ).

Analyses were performed using SPSS (IBM Corp. Released 2020. IBM SPSS Statistics for Windows, Version 27.0. Armonk, NY: IBM Corp). Multiple imputation of missing values was performed using STATA/SE 16.0 (StataCorp. 2019. Stata Statistical Software: Release 16. College Station, TX: StataCorp LLC).

\section{Results}

\section{Participant flow and baseline characteristics}

We randomized 309 persons from June 2018 to July 2019 into gLiFE $(n=153)$ and LiFE $(n=156)$, of which 15 persons dropped out before the start of the intervention. At six months, 44 observations (14.2\%) were lost to followup, $n=25$ in gLiFE (16.3\%) and $n=19$ in LiFE (12.2\%), respectively. Of the envisaged sensor-based 7-day PA measurement, at least 6 full days were completed by 99.0\% of the participants at baseline and $98.8 \%$ at followup. Similarity of baseline values indicates successful randomization (Table 1). The majority of participants was female; on average, participants were cognitively intact, were moderately active, had mediocre motor function, and rather low fear of falling. No study-associated serious adverse events were reported. Of the 3 study-associated adverse events, all had mild consequences: one fall occurred on the way to an assessment (LiFE), one on the way to a gLiFE session, and one during a participant's demonstration of his LiFE execution at home while a trainer was present (LiFE).

\section{Primary outcomes}

\section{Combined endpoint: Falls adjusted for physical activity}

Compared to LiFE, gLiFE had an incidence rate ratio of 1.07 (95\% CI: $0.73 ; 1.57)$ at baseline and of 1.27 (95\% CI: $0.80 ; 2.03)$ at 6 months according to PP analysis. When applying ITT analysis, IRRs at baseline $(1.04 ; 95 \% \mathrm{CI}$ : $0.72 ; 1.50)$ and 6 months $(1.18 ; 95 \%$ CI: $0.75 ; 1.84)$ were smaller (Fig. 2). In both cases, non-inferiority was inconclusive due to upper confidence intervals crossing the $20 \%$ margin $(\Delta)$ at 6 months. This means there was a nonsignificant difference in the risk of experiencing a fall for gLiFE compared to LiFE participants. When subtracting the initial baseline difference of $7.3 \%$ (4.1\%), the changed IRR between both groups remains at 20\% (14\%).
Table 1 Participant characteristics at baseline according to ITT analyses

\begin{tabular}{|c|c|c|c|}
\hline $\mathrm{N}($ mean $\pm \mathrm{SD})$ & $\begin{array}{l}\text { All } \\
N=309\end{array}$ & $\begin{array}{l}\text { LiFE } \\
N=156\end{array}$ & $\begin{array}{l}\text { gLiFE } \\
N=153\end{array}$ \\
\hline Age, years & $78.8 \pm 5.3$ & $78.8 \pm 5.2$ & $78.7 \pm 5.4$ \\
\hline Sex, n (\%) female & $227(73.5)$ & $115(73.7)$ & $112(73.2)$ \\
\hline BMI [kg/m²] & $27.2 \pm 4.9$ & $27.7 \pm 5.0$ & $26.8 \pm 4.7$ \\
\hline No. of medications & $4.9 \pm 3.4$ & $5.0 \pm 3.3$ & $4.8 \pm 3.4$ \\
\hline No. of comorbidities & $2.5 \pm 1.6$ & $2.5 \pm 1.5$ & $2.5 \pm 1.6$ \\
\hline MoCA Score & $26.0 \pm 2.0$ & $26.1 \pm 2.0$ & $25.9 \pm 2.0$ \\
\hline No. of steps/day & $5,659 \pm 2,919$ & $5,778 \pm 3,009$ & $5,538 \pm 2,828$ \\
\hline $\begin{array}{l}\text { No. of falls p.p. in past } \\
6 \text { months }\end{array}$ & $0.66 \pm 1.1$ & $0.66 \pm 1.1$ & $0.65 \pm 1.1$ \\
\hline $\begin{array}{l}\% \text { of fallers in past six } \\
\text { months }\end{array}$ & $126(40.8)$ & $63(40.4)$ & $63(41.2)$ \\
\hline LLFDI Function & $57.3 \pm 7.9$ & $57.4 \pm 8.0$ & $57.3 \pm 7.9$ \\
\hline LLFDI Frequency & $49.4 \pm 4.3$ & $49.3 \pm 4.2$ & $49.5 \pm 4.4$ \\
\hline LLFDI Disability & $70.7 \pm 12.0$ & $71.7 \pm 12.3$ & $69.6 \pm 11.5$ \\
\hline $\begin{array}{l}\text { Gait speed comfortable } \\
{[\mathrm{m} / \mathrm{s}]}\end{array}$ & $1.03 \pm 0.20$ & $1.03 \pm 0.20$ & $1.03 \pm 0.21$ \\
\hline Gait speed fast [m/s] & $1.40 \pm 0.32$ & $1.37 \pm 0.29$ & $1.43 \pm 0.35$ \\
\hline 30 s Chair Stand & $9.1 \pm 3.9$ & $9.2 \pm 3.8$ & $9.0 \pm 3.3$ \\
\hline 8 Level Balance Scale & $4.3 \pm 1.5$ & $4.2 \pm 1.5$ & $4.4 \pm 1.4$ \\
\hline Short FES-I & $10.4 \pm 3.0$ & $10.4 \pm 3.1$ & $10.3 \pm 3.0$ \\
\hline ABC Scale & $75.3 \pm 16.8$ & $75.0 \pm 17.6$ & $75.5 \pm 16.9$ \\
\hline
\end{tabular}

$A B C$ Scale Activities-specific Balance Confidence Scale, BMI body mass index, $C I$ confidence interval, FES-I Falls Efficacy Scale International, ITT intention-to-treat, LLFDI Late Life Function and Disability Instrument, max maximal, MoCA Montreal Cognitive Assessment, No. Number, p.p. per person, SD standard deviation, TUG Timed Up-and-Go

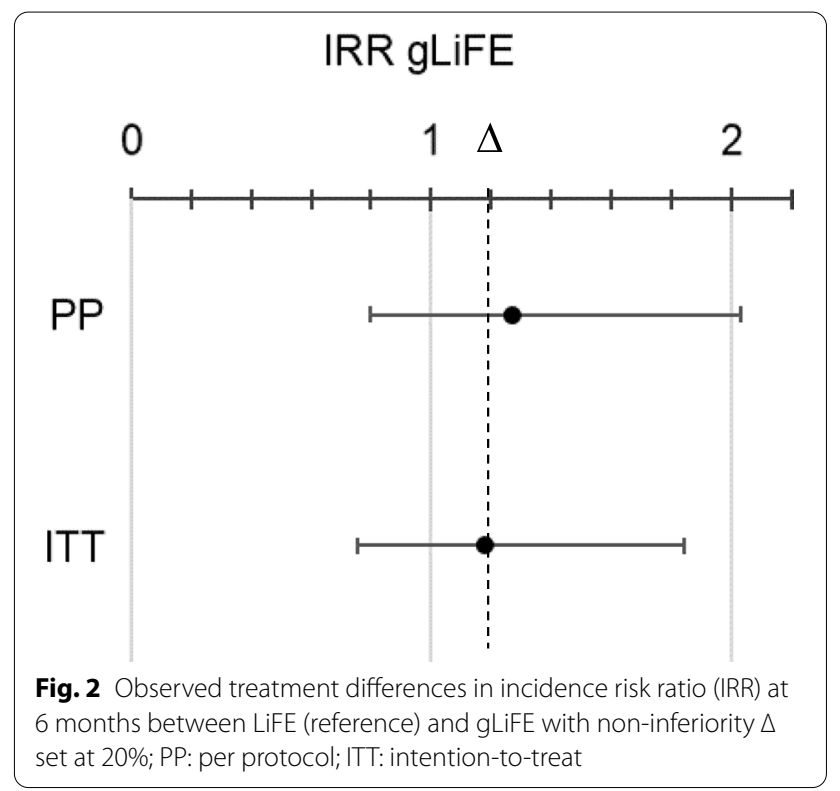




\section{Intervention costs}

Under study conditions the total intervention costs per participant amounted to $€ 350.10$ for LiFE compared to $€ 229.93$ for gLiFE. This corresponded to a cost advantage of $€ 121.17$ for gLiFE. This cost advantage mainly resulted from a difference in personnel costs for trainers. In contrast, costs for room rent and travel expenses were marginally higher for gLiFE than LiFE with $€ 23.45$ and $€ 17.92$, respectively. Under "real world" assumptions, average costs per gLiFE participant were $€ 120.58$ compared to $€ 332.08$ per LiFE participant, resulting in a cost difference of $€ 211.51$ in favour of gLiFE. Again, this was mainly due to a difference in personnel costs. Intervention costs are summarized in table S2.

\section{Secondary outcomes Physical activity}

Both groups increased their amount of steps/day between baseline and follow-up (Table 2). The increase in the gLiFE group was significantly larger than in the LiFE group in both PP (gLiFE to LiFE: +880 steps; CI 252; 1,509) and ITT (gLiFE to LiFE: + 844; CI 176; 1,512) analyses. gLiFE participants increased their steps/day from 5,530 (SE 237); in LiFE, the increase from 5,880 (SE 255) was about a third $(35,5 \%)$ of the increase in the gLiFE group according to ITT (PP: 30,5\%).

\section{Fall outcomes}

According to ITT analyses, 109 falls (PP: 100) were recorded; 49 in LiFE and 60 in gLiFE participants between baseline and 6-month follow-up. Of these, 29 persons fell once in LiFE and 27 in gLiFE; 9 persons in LiFE and 15 in gLiFE fell at least twice (maximum of 4 falls in each group), that is, there were 38 fallers in LiFE and 42 in gLiFE. The incidence of falls per half person year (ITT) in LiFE was 0.30 (SE 0.05) and 0.40 (SE 0.06) in gLiFE (PP; gLiFE: 0.41, SE 0.06; LiFE: 0.30, SE 0.05). The time to event (first fall) and observation time were similar in both groups (median $=180$ days). Comparing the number of falls per half person year at baseline and 6 months, the incidence decreased about 37\% (0.65 to 0.41 ) in gLiFE and 55\% in LiFE (0.66 to 0.30) (ITT). Falls entailed mild (75\%), moderate (18\%), and severe (7\%) consequences as per definition of Schwenk et al. [26].

\section{Motor capacity}

Results of motor capacity assessments are shown in Table 2. No significant differences in changes of motor capacity between groups were found for any of the measures, although improvement in gLiFE was larger in $30 \mathrm{~s}$. chair rise $(+1.00$ vs. +0.38 in LiFE) and 8 level balance scale $(+0.25$ vs. \pm 0.00 in LiFE). LiFE participants showed somewhat larger improvement in comfortable gait speed $(\mathrm{gLiFE}+0.03 \mathrm{~m} / \mathrm{s}$ vs. LiFE $+0.04 \mathrm{~m} / \mathrm{s})$ and fast gait speed (gLiFE -0.02 vs. LiFE +0.02). PP analyses did not alter these findings.

\section{Functional (dis-)ability}

According to LLFDI results, increase in both groups in the functional domain was comparable. Self-perceived function increased slightly from 57.3 (SE 0.6) to 59.2 (SE 0.8) in gLiFE and from 57.4 (SE 0.6) to 58.9 (SE 0.8) (ITT; mean difference 0.3 ; 95\% CI: $-1.33 ; 1.95 ; p=0.710)$. Selfperceived disability improved from 69.6 (SE 0.9) to 71.2 (SE 1.1) in gLiFE and decreased from 71.7 (SE 1.0) to 70.8 (SE 1.0) in LiFE (ITT; mean difference 2.4; 95\% CI: -0.71; $5.50)$ in favor of gLiFE. PP analysis did not alter these findings.

\section{Adherence}

Both groups had a high proportion of completers; $99 \%$ of LiFE and $88 \%$ of gLiFE participants took part in more than $75 \%$ of the sessions. Under ITT stipulations, those numbers expectedly dropped to 91 and $78 \%$, respectively. With 7.8 sessions on average, gLiFE participants attended significantly less sessions than LiFE participants with 8.7 sessions (PP: 95\% CI: -0.68; -1.12). ITT analysis did not change this finding. Duration adherence according to EARS scores were significantly lower in the gLiFE group: 14.9 in gLiFE compared to 16.0 in LiFE (95\% CI: -0.01 ; -2.18). Again, ITT analysis did not affect this finding.

\section{Fear of falling and balance confidence}

Fear of falling decreased in both groups to a comparable level. In gLiFE, it dropped from 10.3 to 9.5 (change of -0.8; SE 0.2); in LiFE, it dropped from 10.4 to 9.6 (change of -0.8; SE 0.2). The between-group difference in this change was not significant (95\% CI: -0.62; 0.62). Balance confidence values (ABC scale) were increased in both groups, however, differences in these changes were not significant $(-2.62 ; 95 \%$ CI: $-6.09 ; 0.84)$. PP analyses did not alter these findings.

\section{Discussion}

In the LiFE-is-LiFE trial it was investigated (1) whether gLiFE as a group format of the LiFE program was noninferior to the individually delivered, original LiFE format in terms of activity-adjusted fall risk, and (2) whether both formats differed in their effect on PA and several function- and adherence-related outcomes. To the best of our knowledge, there has not been any direct comparison of a group format and an individually delivered format of the same intervention program so far; even less one including an economic evaluation. 
Table 2 Baseline and 6-month secondary outcome data and between-group comparison

\begin{tabular}{|c|c|c|c|c|c|c|}
\hline \multirow[t]{2}{*}{ mean $\pm \mathrm{SE}$} & \multicolumn{2}{|l|}{ LiFE } & \multicolumn{2}{|l|}{ gLiFE } & \multicolumn{2}{|c|}{$\begin{array}{l}\text { Between-group difference }(95 \% \mathrm{Cl}) \\
\text { gLiFE vs. LiFE }\end{array}$} \\
\hline & PP & ITT & PP & ITT & PP & ITT \\
\hline \multicolumn{7}{|c|}{ Mean steps/day } \\
\hline Baseline & $5,880 \pm 255$ & $5,778 \pm 250$ & $5,530 \pm 237$ & $5,538 \pm 234$ & & \\
\hline Post & $6,266 \pm 254$ & $6,242 \pm 257$ & $6,797 \pm 264$ & $6,847 \pm 257$ & & \\
\hline Change & $386 \pm 227$ & $465 \pm 257$ & $1,266 \pm 213$ & $1,309 \pm 225$ & $\begin{array}{l}880(252 ; 1,509) \\
p=.007\end{array}$ & $\begin{array}{l}844(176 ; 1,512) \\
p=.015\end{array}$ \\
\hline \multicolumn{7}{|c|}{ LLFDI function } \\
\hline Baseline & $57.4 \pm 0.68$ & $57.4 \pm 0.64$ & $57.4 \pm 0.63$ & $57.3 \pm 0.64$ & & \\
\hline Post & $58.9 \pm 0.75$ & $58.9 \pm 0.76$ & $59.1 \pm 0.76$ & $59.2 \pm 0.77$ & & \\
\hline Change & $1.5 \pm 0.48$ & $1.5 \pm 0.55$ & $1.7 \pm 0.51$ & $1.9 \pm 0.57$ & $\begin{array}{l}0.18(-1.23 ; 1.58) \\
p=.807\end{array}$ & $\begin{array}{l}0.31(-1.33 ; 1.95) \\
p=.710\end{array}$ \\
\hline \multicolumn{7}{|c|}{ LLFDI frequency } \\
\hline Baseline & $49.5 \pm 0.34$ & $49.3 \pm 0.33$ & $49.8 \pm 0.38$ & $49.5 \pm 0.35$ & & \\
\hline Post & $50.3 \pm 0.41$ & $50.1 \pm 0.40$ & $50.2 \pm 0.41$ & $50.0 \pm 0.42$ & & \\
\hline Change & $0.8 \pm 0.29$ & $0.8 \pm 0.30$ & $0.4 \pm 0.30$ & $0.48 \pm 0.34$ & $\begin{array}{l}-0.47(-1.32 ; 0.38) \\
p=.279\end{array}$ & $\begin{array}{l}-0.29(-1.25 ; 0.66) \\
p=.546\end{array}$ \\
\hline \multicolumn{7}{|c|}{ LLFDI disability } \\
\hline Baseline & $71.8 \pm 1.03$ & $71.7 \pm 0.99$ & $69.3 \pm 0.94$ & $69.6 \pm 0.93$ & & \\
\hline Post & $70.8 \pm 1.01$ & $70.8 \pm 1.05$ & $71.3 \pm 1.14$ & $71.2 \pm 1.10$ & & \\
\hline Change & $-1.0 \pm 1.05$ & $-0.9 \pm 1.10$ & $1.9 \pm 1.04$ & $1.5 \pm 1.08$ & $\begin{array}{l}2.87(-0.05 ; 5.79) \\
p=.054\end{array}$ & $\begin{array}{l}2.40(-0.71 ; 5.50) \\
p=.132\end{array}$ \\
\hline \multicolumn{7}{|l|}{ FESI } \\
\hline Baseline & $10.4 \pm 0.27$ & $10.4 \pm 0.25$ & $10.3 \pm 0.25$ & $10.3 \pm 0.24$ & & \\
\hline Post & $9.7 \pm 0.23$ & $9.6 \pm 0.23$ & $9.4 \pm 0.22$ & $9.5 \pm 0.22$ & & \\
\hline Change & $-0.8 \pm 0.24$ & $-0.8 \pm 0.24$ & $-0.9 \pm 0.21$ & $-0.8 \pm 0.22$ & $\begin{array}{l}-0.14(-0.76 ; 0.49) \\
p=.669\end{array}$ & $\begin{array}{l}0.00(-0.62 ; 0.62) \\
p=.996\end{array}$ \\
\hline \multicolumn{7}{|l|}{$A B C$} \\
\hline Baseline & $74.7 \pm 1.48$ & $75.0 \pm 1.41$ & $75.6 \pm 1.36$ & $75.5 \pm 1.36$ & & \\
\hline Post & $78.8 \pm 1.25$ & $79.1 \pm 1.21$ & $77.7 \pm 1.31$ & $77.0 \pm 1.30$ & & \\
\hline Change & $4.1 \pm 1.16$ & $4.1 \pm 1.14$ & $2.1 \pm 1.27$ & $1.5 \pm 1.32$ & $\begin{array}{l}-1.94(-5.31 ; 1.43) \\
p=.259\end{array}$ & $\begin{array}{l}-2.62(-6.09 ; 0.84) \\
p=.138\end{array}$ \\
\hline \multicolumn{7}{|l|}{30 s chair rise } \\
\hline Baseline & $9.1 \pm 0.32$ & $9.2 \pm 0.31$ & $9.0 \pm 0.27$ & $9.0 \pm 0.27$ & & \\
\hline Post & $9.6 \pm 0.36$ & $9.5 \pm 0.34$ & $10.0 \pm 0.38$ & $10.0 \pm 0.40$ & & \\
\hline Change & $0.49 \pm 0.31$ & $0.38 \pm 0.30$ & $1.10 \pm 0.31$ & $1.00 \pm 0.34$ & $\begin{array}{l}0.56(-0.26 ; 1.38) \\
p=.180\end{array}$ & $\begin{array}{l}0.63(-0.25 ; 1.51) \\
p=.164\end{array}$ \\
\hline \multicolumn{7}{|c|}{8 level balance scale } \\
\hline Baseline & $4.2 \pm 0.13$ & $4.2 \pm 0.12$ & $4.4 \pm 0.12$ & $4.4 \pm 0.11$ & & \\
\hline Post & $4.2 \pm 0.13$ & $4.2 \pm 0.13$ & $4.6 \pm 0.14$ & $4.6 \pm 0.14$ & & \\
\hline Change & $0.0 \pm 0.12$ & $0.0 \pm 0.13$ & $0.2 \pm 0.14$ & $0.25 \pm 0.13$ & $\begin{array}{l}0.21(-0.58 ; 0.15) \\
p=.248\end{array}$ & $\begin{array}{l}0.20(-0.17 ; 0.58) \\
p=.290\end{array}$ \\
\hline \multicolumn{7}{|c|}{ Gait speed normal } \\
\hline Baseline & $1.04 \pm 0.02$ & $1.03 \pm 0.02$ & $1.05 \pm 0.02$ & $1.03 \pm 0.02$ & & \\
\hline Post & $1.07 \pm 0.02$ & $1.07 \pm 0.02$ & $1.07 \pm 0.02$ & $1.06 \pm 0.02$ & & \\
\hline Change & $0.04 \pm 0.01$ & $0.04 \pm 0.01$ & $0.02 \pm 0.02$ & $0.03 \pm 0.02$ & $\begin{array}{l}-0.01(-0.05 ; 0.02) \\
p=.458\end{array}$ & $\begin{array}{l}-0.01(-0.05 ; 0.03) \\
p=.651\end{array}$ \\
\hline \multicolumn{7}{|c|}{ Gait speed fast } \\
\hline Baseline & $1.38 \pm 0.02$ & $1.37 \pm 0.02$ & $1.44 \pm 0.03$ & $1.43 \pm 0.03$ & & \\
\hline Post & $1.40 \pm 0.03$ & $1.39 \pm 0.03$ & $1.43 \pm 0.03$ & $1.41 \pm 0.03$ & & \\
\hline Change & $0.02 \pm 0.02$ & $0.02 \pm 0.02$ & $-0.01 \pm 0.02$ & $-0.02 \pm 0.02$ & $\begin{array}{l}-0.03(-0.08 ; 0.02) \\
p=.243\end{array}$ & $\begin{array}{l}-0.04(-0.09 ; 0.02) \\
p=.212\end{array}$ \\
\hline
\end{tabular}




\section{Primary outcomes}

For the main outcome, activity-adjusted fall risk operationalized as IRR, non-inferiority was not confirmed as the upper bound of the two-sided 95\% confidence interval crossed the predefined $20 \%$ non-inferiority margin. Per definition, this means that non-inferiority of gLiFE is 'inconclusive' [18]. One reason for this could be that we used 6-month instead of 12-month data, and that differences at 6 months are of different nature than at 12 months. As there are less fall events within 6 than within 12 months, there is a higher risk of random error which could have influenced the point estimate. From an intervention perspective, the LiFE group was under more direct and closer individual supervision than gLiFE participants during the intervention phase, which might come with more problems to independently conduct and adapt LiFE activities as compared to gLiFE participants in the long term. Unlike LiFE participants who had direct suggestions and support, gLiFE participants had learnt to implement LiFE activities in their own way at home right from the start. We also see that the mean IRR is very close to the $20 \%$ margin, suggesting that the actual difference could be close to these $20 \%$.

Comparing the reduction in overall incidence of falls between baseline and follow-up, both groups in the present trial reduced their fall incidence to a great extent. Despite the fact that fall incidence was already low in our sample at baseline, these reductions were greater $(37 \%$ gLiFE; $55 \%$ LiFE) than in the LiFE group in the reference trial by Clemson et al. (22\%) [14].

Regarding the second primary outcome, intervention costs of several exercise-based fall prevention programs have already been determined as part of economic analyses [35]. However, such economic analyses have not been performed for the LiFE program, yet, despite the high effectivity of this program for reducing falls and increasing PA [14]. This is of interest for potential payers of the intervention in case of implementation. Our findings highlight that gLiFE was associated with lower intervention costs compared to LiFE while at the same time reducing falls in both formats, making it an attractive alternative from a payer's and individual's perspective. The size of the cost advantage depended primarily on the ratio of participants to trainers. Therefore, the cost advantage was particularly pronounced in the "real world" scenario, which assumed a higher number of participants per group. Moreover, the total intervention costs per participant depended on the trainers' salary or the number of groups each trainer/trainer pair supervises. Hence, there is not only one possible scenario of the "real world", but the assumptions made in this study were found to be the most realistic by intervention experts. For an informed recommendation regarding implementation, other health-care utilization costs beside intervention costs must be examined in relation to the health effects. An economic evaluation regarding the cost-effectiveness of gLiFE will assist in making implementation recommendations and is part of further analyses [36].

\section{Secondary outcomes}

In general, the main idea of the LiFE program-to promote safe PA and simultaneously improve motor function [15]-was confirmed. PA, operationalized as walking activity for our study purpose, was increased in both groups. With $23 \%$, this increase was significantly higher in gLiFE than in LiFE with 7\%. Walking has been identified as key factor in promoting PA and health [37] and steps/day are a tangible activity goal for both participants and trainers [38]. Increased walking activity over more than 1,000 steps/day comes with lower risk of all-cause mortality as well as cardiovascular disease morbidity and mortality [39]. Hence, on average gLiFE seems better suited to evoke activity-related health benefits. This is further supported in that other studies have shown much lower pre-to-post intervention changes in steps/day, averaging around 800 steps change in older, mainly community-dwelling adults [40]. With an average between 5,500 and 5,800 steps/day in both groups at baseline, our sample was slightly more active than in studies with large samples of men and women of similar age [7, 41], indicating at least moderate activity levels. The examination of mechanisms of action in LiFE and gLiFE do not provide evidence for the superiority of gLiFE in affecting psychological determinants which are assumed to translate into behavioral changes [42]. However, it is possible that gLiFE participants profited of the group program in a way we did not capture with our measurements, e.g., through comparison with peers.

For other secondary outcomes, there were no significant differences in changes over six months between both groups. Descriptive data showed somewhat larger improvement over 6 months in gLiFE for LLFDI disability, but not for any other secondary measure. The difference between comfortable and fast gait speed at baseline indicates that there is a notable reserve in functional capacity in our sample. Taking into account that gLiFE participants had significantly lower attendance rates and duration adherence, it seems that the 'dose' given to gLiFE participants was still sufficient to achieve effects comparable to LiFE.

The LiFE intervention was delivered similarly to the reference trial by Clemson and colleagues [14], but underwent small organizational changes, which were needed to align LiFE and gLiFE contents in our study (this is further discussed elsewhere [17, 19]). Duration adherence at 6 months according to EARS was medium to good 
in both groups with mean values confirming adherence to their plans. LiFE participants had a higher adherence at 6 months, which could be due to higher intervention attendance rates. The fact that attendance was significantly higher in LiFE than in gLiFE was not surprising as the group session schedule was predetermined and not movable whereas individual appointments in LiFE could easily be moved according to participants' requirements. One factor that might have boosted effectiveness of gLiFE is social support by other group members, which was found to be supportive of engagement in exercise and PA [43]. According to an extensive review on exercise interventions to prevent falls, however, there is no difference in effect based on intervention format (group vs. individual) [44]. Further analyses are needed to determine which characteristics of both formats are responsible for differences in both groups' outcomes.

\section{Strengths and limitations}

Results of the present study need to be interpreted in the light of several strengths and limitations. Non-inferiority trials are becoming more frequent, aiming to establish interventions' non-inferiority over another treatment [19]. Instead of developing new interventions which then have to undergo extensive scientific evaluation, it seems worth looking at already established intervention programs such as LiFE. By adapting or refining existing interventions, their feasibility and cost-effectiveness could be improved, which in turn would come with advantages for participants and payers equally. The LiFE program has the benefit of being carried out at participants' homes, which entails fewer burdens to physical exercise than conventionally delivered structured exercise. Many of those burdens, especially those being highlighted by older adults [45] do not apply in the LiFE program (e.g., bad weather, lack of time). Having shown that both modes of delivery come with meaningful health benefits such as enhanced PA, gLiFE could now be made available also to those who prefer company of others. At the same time, those who prefer being on their own can be served as well. Another strength is that activityadjusted falls were assessed using highly reliable methods. For fall documentation, participants completed fall calendars [46]; PA was assessed using highly reliable sensors over a full week with very few incomplete measurements $(<2 \%)$. Moreover, we followed the extended consort statement of 2010 for non-inferiority trials, thus abiding by clear reporting and interpretation standards. Lastly, data analyses were carried out for both PP and ITT including multiple imputation [32].

Despite many strengths, some limitations are to be considered. As pandemic circumstances had a strong impact on older adults' habitual PA and overall movement behavior [47] it is expected that 12-month follow-up data were highly biased. COVID-19 pandemic regulations began shortly after completion of the 6-month assessments, and about one third of the participants were not assessed regularly as part of 12-month follow-up within the specified time window. Attempts were therefore made to follow up any unscheduled assessment after reopening of public structures following the lockdown. Therefore, we chose to deviate from the study protocol by evaluating non-inferiority based on 6-month instead of 12-month data. Moreover, pre-baseline falls data were assessed retrospectively for 6 months. Comparing 6-month follow-up fall data with baseline falls therefore is to be done very cautiously due to the different standard and sources of bias in falls assessment [24]. Due to the established effectiveness of the LiFE program [14], no control group was included in this trial. Natural progression of IRRs without intervention therefore cannot be quantified. Compared to the Australian LiFE study [14], the present sample was somewhat younger ( -4 years), had a higher proportion of women $(+14 \%)$ and less falls in the past (0.66 per person and half year compared to 2.13 per person and year in Clemson et al.), which limits comparability with our findings.

\section{Conclusions}

Non-inferiority of gLiFE's reduction of falls compared to LiFE was inconclusive, while its increase in walking activity was significantly higher than in LiFE, which shows its large potential especially in promoting PA. In the light of lower intervention costs compared to LiFE, gLiFE is an alternative from a payer's and individual's perspective. Our results suggest that both formats come with important effects and advantages, and that individuals should be given the opportunity to choose between both formats depending on their individual goals.

\begin{abstract}
Abbreviations
ABC Scale: Activities-specific balance confidence scale; BMI: Body mass index; Cl: Confidence interval; EARS: Exercise Adherence Rating Scale; FES-I: Falls Efficacy Scale International; gLiFE: Group-based Lifestyle-integrated Functional Exercise; IRR: Incidence rate ratio; ITT: Intention-to-treat; LiFE: Lifestyle-integrated Functional Exercise; LLFDI: Late-life function and disability instrument; MoCA: Montreal cognitive assessment; No.: Number; PA: Physical activity; p.p.: Per person; PP: Per protocol; SD: Standard deviation; SE: Standard error; TIDieR: Template for intervention description and replication; TUG: Timed Up-and-Go test.
\end{abstract}

\section{Supplementary Information}

The online version contains supplementary material available at https://doi. org/10.1186/s12966-021-01190-z.

Additional file 1. Data and assumptions for the calculation of Intervention costs by scenario 
Additional file 2. Intervention costs per participant for gLiFE and LiFE by scenario and cost category

Additional file 3. CONSORT Statement 2006 - Checklist for Non-inferiority and Equivalence Trials

\section{Acknowledgements}

We thank Christoph Endress, Julia Gugenhan, Anna Kroog, Birgit Och, Malte Liebl-Wachsmuth, Mona Bär, Martin Bongartz, and Annette Lohmann for their invaluable support in carrying out this study.

\section{Authors' contributions}

$\mathrm{CPJ}, \mathrm{CN}, \mathrm{SL}, \mathrm{JK}, \mathrm{JD}, \mathrm{HHK}, \mathrm{LC}, \mathrm{CB}$, and MS have made substantial contributions to the conception and design of this work; CPJ, CN, FKG, and SL have carried out data acquisition; CPJ, SL, SG, JK, JD, and MS have contributed to data preparation and analyses; all authors have contributed to interpretation of data and have drafted and revised this work substantially; all authors have approved the submitted version; all authors have agreed both to be personally accountable for the author's own contributions and to ensure that questions related to the accuracy or integrity of any part of the work, even ones in which the author was not personally involved, are appropriately investigated, resolved, and the resolution documented in the literature.

\section{Funding}

This work was supported by the German Federal Ministry of Education and Research [grant number 01GL1705A-D]. The funders had no role in study concept and design, data collection, analysis and interpretation, or the preparation and the decision to submit this manuscript for publication. Open Access funding enabled and organized by Projekt DEAL.

\section{Availability of data and materials}

The datasets used and/or analyzed during the current study are available from the corresponding author on reasonable request.

\section{Declarations}

\section{Ethics approval and consent to participate}

Ethical approval was obtained for both sites: Heidelberg document number Schwe2017 2/1-1; Stuttgart document number 723/2017BO2. All participants gave written informed consent prior to participation The study is conforming to the respective policy and mandates of the Declaration of Helsinki.

\section{Consent for publication}

Not applicable.

\section{Competing interests}

The authors declare that they have no competing interests.

\section{Author details}

${ }^{1}$ Network Aging Research, Heidelberg University, Bergheimer Strasse 20, 69115 Heidelberg, Germany. ${ }^{2}$ Department of Clinical Gerontology and Geriatric Rehabilitation, Robert Bosch Hospital, Stuttgart, Germany. ${ }^{3}$ Department of Health Economics and Health Services Research, University Medical Center Hamburg-Eppendorf, Hamburg, Germany. ${ }^{4}$ Institute of Epidemiology and Medical Biometry, Ulm University, Ulm, Germany. ${ }^{5} \mathrm{~B}$ University of Health and Social Sciences, Study Centre Stuttgart, Stuttgart, Germany. ${ }^{6}$ Faculty of Health Sciences, University of Sydney, Sydney, Australia.

Received: 27 April 2021 Accepted: 23 August 2021

Published online: 03 September 2021

\section{References}

1. Bauman A, Merom D, Bull FC, Buchner DM, Fiatarone Singh MA. Updating the evidence for physical activity: summative reviews of the epidemiological evidence, prevalence, and interventions to promote "active aging." Gerontologist. 2016;56(Suppl 2):S268-80.
2. Reiner M, Niermann C, Jekauc D, Woll A. Long-term health benefits of physical activity-a systematic review of longitudinal studies. BMC Public Health. 2013;13:813.

3. Crimmins EM. Lifespan and healthspan: past, present, and promise. Gerontologist. 2015;55(6):901-11.

4. Nelson ME, Rejeski WJ, Blair SN, Duncan PW, Judge JO, King AC, et al. Physical activity and public health in older adults: recommendation from the American College of Sports Medicine and the American Heart Association. Med Sci Sports Exerc. 2007;39(8):1435-45.

5. Mertz KJ, Lee DC, Sui X, Powell KE, Blair SN. Falls among adults: the association of cardiorespiratory fitness and physical activity with walking-related falls. Am J Prev Med. 2010;39(1):15-24.

6. Lawton BA, Rose SB, Elley CR, Dowell AC, Fenton A, Moyes SA. Exercise on prescription for women aged 40-74 recruited through primary care: two year randomised controlled trial. BMJ. 2008;337:a2509.

7. Jefferis BJ, Merom D, Sartini C, Wannamethee SG, Ash S, Lennon LT, et al. Physical activity and falls in older men: the critical role of mobility limitations. Med Sci Sports Exerc. 2015;47(10):2119-28.

8. Gregg EW, Pereira MA, Caspersen CJ. Physical activity, falls, and fractures among older adults: a review of the epidemiologic evidence. J Am Geriatr Soc. 2000;48(8):883-93.

9. Tinetti ME, Speechley M, Ginter SF. Risk factors for falls among elderly persons living in the community. N Engl J Med. 1988;319(26):1701-7.

10. Berg WP, Alessio HM, Mills EM, Tong C. Circumstances and consequences of falls in independent community-dwelling older adults. Age Ageing. 1997;26(4):261-8.

11. Del Din S, Galna B, Lord S, Nieuwboer A, Bekkers EMJ, Pelosin E, et al. Falls risk in relation to activity exposure in high-risk older adults. J Gerontol A Biol Sci Med Sci. 2020;75(6):1198-205.

12. Klenk J, Kerse N, Rapp K, Nikolaus T, Becker C, Rothenbacher D, et al. Physical activity and different concepts of fall risk estimation in older people-results of the ActiFE-UIm Study. PloS one. 2015;10(6):e0129098.

13. Wijlhuizen GJ, Chorus AM, Hopman-Rock M. The FARE: a new way to express FAlls Risk among older persons including physical activity as a measure of exposure. Prev Med. 2010:50(3):143-7.

14. Clemson L, Fiatarone Singh MA, Bundy A, Cumming RG, Manollaras K, $\mathrm{O}^{\prime}$ Loughlin $\mathrm{P}$, et al. Integration of balance and strength training into daily life activity to reduce rate of falls in older people (the LiFE study): randomised parallel trial. BMJ. 2012;345:e4547.

15. Clemson L, Munro J, Fiatarone Singh MA. Lifestyle-integrated Functional Exercise (LiFE) program to prevent falls. Trainer's manual. Sydney: Sydney University Press; 2014.

16. Li K, Comer K, Huang T, Schmidt K, Tong M. Effectiveness of a modified lifestyle-integrated functional exercise program in residential retirement communities - a pilot study. SAGE Open Nursing. 2018:4:2377960818793033.

17. Kramer F, Labudek S, Jansen CP, Nerz C, Fleig L, Clemson L, et al. Development of a conceptual framework for a group-based format of the Lifestyle-integrated Functional Exercise (gLiFE) programme and its initial feasibility testing. Pilot Feasibility Stud. 2020;6:6.

18. Piaggio G, Elbourne DR, Pocock SJ, Evans SJ, Altman DG. Reporting of noninferiority and equivalence randomized trials: extension of the CONSORT 2010 statement. JAMA. 2012;308(24):2594-604.

19. Jansen CP, Nerz C, Kramer F, Labudek S, Klenk J, Dams J, et al. Comparison of a group-delivered and individually delivered lifestyle-integrated functional exercise (LiFE) program in older persons: a randomized noninferiority trial. BMC Geriatr. 2018;18(1):267.

20. Podsiadlo D, Richardson S. The timed "Up \& Go": a test of basic functional mobility for frail elderly persons. J Am Geriatr Soc. 1991;39(2):142-8.

21. Moore GF, Audrey S, Barker M, Bond L, Bonell C, Hardeman W, et al. Process evaluation of complex interventions: Medical Research Council guidance. BMJ. 2015;350:h1258.

22. Ryan CG, Grant PM, Tigbe WW, Granat MH. The validity and reliability of a novel activity monitor as a measure of walking. Br J Sports Med. 2006:40(9):779-84.

23. Klenk J, Peter RS, Rapp K, Dallmeier D, Rothenbacher D, Denkinger M, et al. Lazy Sundays: role of day of the week and reactivity on objectively measured physical activity in older people. Eur Rev Aging Phys Act. 2019;16:18. 
24. Lamb SE, Jorstad-Stein EC, Hauer K, Becker C. Development of a common outcome data set for fall injury prevention trials: the Prevention of Falls Network Europe consensus. J Am Geriatr Soc. 2005;53(9):1618-22.

25 Gillespie LD, Robertson MC, Gillespie WJ, Sherrington C, Gates S, Clemson LM, et al. Interventions for preventing falls in older people living in the community. Cochrane Database Syst Rev. 2012;2021(9):Cd007146.

26. Schwenk M, Lauenroth A, Stock C, Moreno RR, Oster P, McHugh G, et al. Definitions and methods of measuring and reporting on injurious falls in randomised controlled fall prevention trials: a systematic review. BMC Med Res Methodol. 2012;12:50.

27. Jones CJ, Rikli RE, Beam WC. A 30-s chair-stand test as a measure of lower body strength in community-residing older adults. Res Q Exerc Sport. 1999;70(2):113-9.

28. Hawley-Hague H, Horne M, Skelton DA, Todd C. Review of how we should define (and measure) adherence in studies examining older adults' participation in exercise classes. BMJ Open. 2016;6(6):e011560.

29. Newman-Beinart NA, Norton S, Dowling D, Gavriloff D, Vari C, Weinman $J A$, et al. The development and initial psychometric evaluation of a measure assessing adherence to prescribed exercise: the Exercise Adherence Rating Scale (EARS). Physiotherapy. 2017;103(2):180-5.

30. Kempen GI, Yardley L, van Haastregt JC, Zijlstra GA, Beyer N, Hauer K, et al. The Short FES-I: a shortened version of the falls efficacy scale-international to assess fear of falling. Age Ageing. 2008;37(1):45-50.

31. European Agency for the Evaluation of Medicinal Products. Points to cconsider on witching between superiority and non-inferiority. In: (CPMP) CfPMP, editor. London: European Agency for the Evaluation of Medicinal Products; 2000.

32. Dong Y, Peng CY. Principled missing data methods for researchers. Springerplus. 2013;2(1):222.

33. Azur MJ, Stuart EA, Frangakis C, Leaf PJ. Multiple imputation by chained equations: what is it and how does it work? Int J Methods Psychiatr Res. 2011;20(1):40-9.

34. Little R, Rubin D. Statistical analysis with missing data. 3rd ed. Hoboken: Wiley; 2019

35. Winser SJ, Chan HTF, Ho L, Chung LS, Ching LT, Felix TKL, et al. Dosage for cost-effective exercise-based falls prevention programs for older people: A systematic review of economic evaluations. Ann Phys Rehabil Med. 2020;63(1):69-80.

36. Gottschalk S, König H, Schwenk M, Nerz C, Becker C, Klenk J, et al. Costeffectiveness of a group- versus individually-delivered exercise program in community-dwelling persons aged 70+. J Am Med Dir Assoc. (under review).

37. Office of the Surgeon G. Publications and Reports of the Surgeon General. Step It Up! The Surgeon General's call to action to promote walking and walkable communities. Washington (DC): US Department of Health and Human Services; 2015.

38. Kraus WE, Janz KF, Powell KE, Campbell WW, Jakicic JM, Troiano RP, et al. Daily step counts for measuring physical activity exposure and its relation to health. Med Sci Sports Exerc. 2019;51(6):1206-12.

39. Hall KS, Hyde ET, Bassett DR, Carlson SA, Carnethon MR, Ekelund U, et al. Systematic review of the prospective association of daily step counts with risk of mortality, cardiovascular disease, and dysglycemia. Int J Behav Nutr Phys Act. 2020;17(1):78

40. Tudor-Locke C, Craig CL, Aoyagi Y, Bell RC, Croteau KA, De Bourdeaudhuij I, et al. How many steps/day are enough? For older adults and special populations. Int J Behav Nutr Phys Act. 2011;8(1):80.

41. Lee IM, Shiroma EJ, Kamada M, Bassett DR, Matthews CE, Buring JE. Association of step volume and intensity with all-cause mortality in older women. JAMA Intern Med. 2019;179:1105.

42. Labudek S, Fleig L, Jansen C-P, Kramer-Gmeiner F, Nerz C, Clemson L, et al. Effects of lifestyle-integrated fall prevention on psychological determinants of behavior change (in preparation).

43. Haughton MCNeill L, Wyrwich KW, Brownson RC, Clark EM, Kreuter MW. Individual, social environmental, and physical environmental influences on physical activity among black and white Adults: A structural equation analysis. Ann Behav Med. 2006;31(1):36-44.

44. Sherrington C, Fairhall N, Kwok W, Wallbank G, Tiedemann A, Michaleff ZA, et al. Evidence on physical activity and falls prevention for people aged 65+ years: systematic review to inform the WHO guidelines on physical activity and sedentary behaviour. Int J Behav Nutr Phys Act. 2020;17(1):144.
45. Moschny A, Platen P, Klaaßen-Mielke R, Trampisch U, Hinrichs T. Barriers to physical activity in older adults in Germany: a cross-sectional study. Int J Behav Nutr Phys Act. 2011;8(1):121.

46. Hauer K, Lamb SE, Jorstad EC, Todd C, Becker C. Systematic review of definitions and methods of measuring falls in randomised controlled fall prevention trials. Age Ageing. 2006;35(1):5-10.

47. Ammar A, Brach M, Trabelsi K, Chtourou H, Boukhris O, Masmoudi L, et al. Effects of COVID-19 home confinement on eating behaviour and physical activity: Results of the ECLB-COVID19 International Online Survey. Nutrients. 2020;12(6):1583.

\section{Publisher's Note}

Springer Nature remains neutral with regard to jurisdictional claims in published maps and institutional affiliations.

Ready to submit your research? Choose BMC and benefit from:

- fast, convenient online submission

- thorough peer review by experienced researchers in your field

- rapid publication on acceptance

- support for research data, including large and complex data types

- gold Open Access which fosters wider collaboration and increased citations

- maximum visibility for your research: over 100M website views per year

At $\mathrm{BMC}$, research is always in progress.

Learn more biomedcentral.com/submissions 